\title{
Démographie, famille et reproduction familiale : un dialogue en
} évolution

Luigi Lorenzetti, Muriel Neven

\section{Citer ce document / Cite this document :}

Lorenzetti Luigi, Neven Muriel. Démographie, famille et reproduction familiale : un dialogue en évolution. In: Annales de démographie historique, 2000-2. Famille et parenté. pp. 83-100;

doi : 10.3406/adh.2001.1976

http://www.persee.fr/doc/adh_0066-2062_2001_num_2000_2_1976

Document généré le 28/07/2017 


\begin{abstract}
The most recent trends of historical research relating to family reproduction sheds light on the progressive elaboration of an interdisciplinary dialogue which mixes individuals', families' and communities time. During the last few years, historical demography on the one hand, family history and ethnology on the other hand, have built still narrower links. The first one concentrated itself on the influence of demographic processes on family forms ; the second ones tried to illustrate the impact of family system on demographic dynamics. The results largely illustrated the variety of solutions put into effect by families to ensure their reproduction. Far from being a limit for the interpretation possibilities of family Systems, this variety represents on the contrary a real wealth and this even more since new methods, among which event history analysis, should make it possible to estimate the impact of short run on strategies on the long run at the family level, Le. those of transmission between generations.
\end{abstract}

\title{
Résumé
}

Les tendances les plus récentes de la recherche historique relatives à la reproduction familiale mettent en évidence l'élaboration progressive d'un dialogue interdisciplinaire qui croise les temps des individus, des familles et des communautés. Au cours des dernières années, la démographie historique d'une part, l'histoire de la famille et l'ethnologie d'autre part, ont tissé des liens de plus en plus étroits. La première a concentré son regard sur l'influence des processus démographiques sur les formes familiales, les deuxièmes se sont efforcées d'illustrer l'impact des systèmes familiaux sur les dynamiques démographiques. Les résultats ont amplement illustré la variété des solutions mises en œuvre par les familles pour assurer leur reproduction. Loin de constituer une limite aux possibilités d'interprétation des systèmes familiaux, cette variété représente, au contraire, une véritable richesse, d'autant que l'apport de méthodes nouvelles, parmi lesquelles l'Event History Analysis devrait permettre de mieux évaluer l'impact du court terme, de l'accidentel, sur les stratégies de long terme à l'échelle familiale, c'est-à-dire celles de la transmission entre générations. 


\title{
DÉMOGRAPHIE, FAMILLE ET REPRODUCTION FAMILIALE: UN DIALOGUE EN ÉVOLUTION
}

\author{
par Luigi LORENZETTI et Muriel NEVEN
}

Résumer en quelques pages de nombreuses années de recherches dans le domaine de l'histoire de la famille et de la reproduction familiale représente un défi presque insurmontable étant donné la complexité du sujet et l'importance des réflexions qui en sont issues. Les pages qui suivent ne prétendent en aucun cas être exhaustives; plus modestement, elles espèrent aborder les nombreuses questions que les historiens démographes et les historiens de la famille ont soulevées au cours des dernières années et mettre en évidence les tendances les plus récentes de la recherche historique sur la reproduction familiale. Au sein d'un bilan sur une discipline, la démographie historique, qui se perçoit encore parfois en crise, notre espoir est de montrer la fécondité du travail interdisciplinaire qui se construit depuis plusieurs années sur la complexité et les croisements des temps des individus, des familles et des communautés.

\section{UN DIALOGUE \\ INTERDISCIPLINAIRE DIFFICIIE}

Depuis que l'histoire est née comme discipline scientifique, les histoires du droit traitent des normes d'héritage. Pour étudier la reproduction familiale, des approches conjointes des réalités démographiques et des coutumes du droit en matière d'héritage, de succession, de transmission s'avèrent nécessaires. Il est bien évident que les historiens de la population et les historiens du droit ne sont pas spontanément les mêmes personnes dans la tradition académique classique. Cependant, l'historiographie présente plusieurs points de contacts, façonnés par des chercheurs qui ne se définissent pas comme des historiens-démographes.

En croisant histoire de la famille et histoire de la transmission, Yver (1966), Le Roy Ladurie (1972), Goody (1976) ou Burguière (1986) ont développé l'anthropologie historique (pour une synthèse, voir Burguière, 1995). Ils se spécifient par une approche très centrée sur l'espace, l'identification de frontières comme la fameuse ligne Genève-SaintMalo, censée séparer " les deux France qui viennent là se rejoindre. De part et d'autre de la diagonale qui coupe en deux le territoire, tout semble différer, les paysages agraires comme les technologies, la densité des communications comme celle des manufactures, la taille des hommes comme leur aptitude à maîtriser l'écrit " (Chartier, 1992, 739). Quand, dès 1972, Emmanuel Le Roy Ladurie divise en trois zones l'espace français sur la base des "Structures familiales et coutumes d'héritage ", il tente explicitement d'interpréter des règles juridiques en termes de systèmes familiaux, ce qui, 
outre une indéniable fascination de l'espace, est la deuxième dimension de cette démarche intellectuelle et méthodologique (Brennan, James, Morill, 1982; pour une revue critique, voir Oris, Ochiai, 2000).

Un second courant s'est épanoui à partir du Groupe de Cambridge et des travaux pionniers de Laslett ou Wall (1972). Il aborde l'histoire de la famille par le biais de l'organisation du groupe domestique. La fameuse typologie des ménages élaborée par Laslett a d'emblée mis en exergue l'existence d'une diversité dans les formes familiales bien avant la révolution industrielle: elle est devenue une référence presque incontournable dès qu'un chercheur s'attache à décrire les structures du foyer. Comme les anthropologues, ces historiens de la famille découpent l'espace en grandes régions culturelles, en fonction cette fois des types de ménages dominants dans les sociétés étudiées. Hajnal (1983) ou Laslett (1983) ont ainsi proposé des cartographies des systèmes familiaux en Europe, qui se fondent sur des critères pour le moins divergents (âge au mariage, structure du ménage, organisation du travail au sein du groupe domestique, circulation des servantes, etc.), mais qui à nouveau grossissent le tracé des frontières comme la célèbre ligne Trieste-Saint-Petersbourg entre les systèmes familiaux occidentaux et l'immense Orient (pour un aperçu à la fois succinct et complet, voir Oris, Ochiai, 2000, tableau 1).

Dans le prolongement de l'essai de Burguière (1986), la tentative de synthèse la plus ambitieuse entre l'anthropologie historique de la famille et l'analyse des structures du groupe domestique fut réalisée par Emmanuel Todd. Découpant l'espace européen
(1990), puis même mondial (1999), en régions caractérisées par l'association de formes domestiques, de modes de transmission et de structures agraires, il fait œuvre originale en intégrant à un niveau macro-économique une dimension fondamentale de la vie des sociétés préindustrielles, les modes d'exploitation du sol. Il va même plus loin en utilisant cette grille spatiale pour lire l'evolution politique de l'Europe et du monde. La synthèse de Todd, à beaucoup d'égards, est le couronnement naturel de toute une orientation de recherches, même si elle a fait l'objet de nombreuses critiques. Au-delà des polémiques sur ses interprétations, deux problèmes majeurs sont à souligner, problèmes qui sont davantage imputables à un courant de pensée qu'à un auteur en particulier: (1) la non-prise en compte de la diversité - plus précisément de la spécificité des environnements - et (2) l'intégration insuffisante de la notion de temps, à la fois individuel et familial.

Les typologies d'Hajnal, de Laslett ou de Todd ont été largement critiquées en raison de leur caractère réducteur. $A$ l'intérieur de chacune de leurs grandes régions, la multiplication des études de cas a mis en évidence la diversité et non l'homogénéité. Notamment des différences notables entre monde urbain et rural ne trouvent pas leur place dans les visions macro-spatiales, pas plus que la différenciation des types familiaux en fonction du statut social ${ }^{1}$. Le dernier état de la question, celui d'Antoinette Fauve-Chamoux et de Richard Wall (1997), s'assimilait d'ailleurs à une sorte de renonciation devant un savoir éclaté qui défie les synthèses.

Quant à la critique relative à l'a-temporalité, elle repose en réalité sur trois arguments majeurs. D'abord, des auteurs 
comme Laslett (Laslett, 1983, 558), Burguière (1986) ou Todd (1990) assument de manière explicite la longue durée des systèmes familiaux. Or, plusieurs contre-exemples mettent au contraire en lumière leur évolution, leurs adaptations en réponse aux pressions externes ou internes, le plus célèbre est celui de la famille nucléaire irlandaise qui, après la grande Famine, devient non-égalitaire et de type "famille souche ", entraînant l'émigration transcontinentale des cadets (Oris, Ochiai, 2000, 6; Fauve-Chamoux, Wall, 1997; Derouet, 1992). D'autre part, et ce deuxième volet de la critique est bien connu, l'étude de la structure des ménages à partir de recensements cache le passage des groupes domestiques par des phases de complexité (Berkner, 1972, 1975; Janssens, 1993, 10-13) er, par ailleurs, accorde une primauté exagérée à la lecture statistique de typologies établies rétrospectivement par les historiens, au détriment des idéaux familiaux qui dominaient dans les sociétés du passé (Neven, 2000a, 34, 214; Derouet, 1998). C'est la prise de conscience de cette limite qui a suscité la mise au point de systèmes de simulations du type CAMSIN, qui permettent de voir dans quelle mesure le régime démographique d'une société donnée produit de façon mécanique une simplicité factice en ce sens qu'elle ne reflète pas nécessairement les idéaux familiaux (Reher, 1997, 248 sq; Ruggles, 1996; Perrenoud, 1998; Devolder, 2000). Une autre réaction a été, à travers de patients travaux de couplage, de multiplier les études longitudinales (voir par exemple le volume de FauveChamoux et Ochiai, 1998). En effet, il est discutable de raisonner en termes de systèmes familiaux, donc de fonctionnement, en se fondant sur l'observation de structures.
Nous ne nous étendrons pas sur cet aspect, car c'est un des points que nous essayons de développer dans les sections suivantes, moins orientées vers la critique et davantage tournées vers les nouvelles questions et les nouvelles méthodes. Soulignons toutefois que les historiens ont souvent fait un procès d'intention au groupe de Cambridge, en lui attribuant des intentions qui n'étaient pas les siennes au départ. Les recherches rassemblées par $\mathrm{R}$. Smith (1984) ou l'élaboration de la nuclear hardship hypothesis (Laslett, 1988) attestent d'un travail de fond qui dépasse largement les clichés structuralistes. Les travaux sur le life cycle servanthood ont aussi nettement contribué à une meilleure compréhension du fonctionnement du modèle européen de mariage tardif (Wall, 1978; Segalen, 1985; Guinanne, 1992b).

En fait, sans renier les acquis scientifiques de ces recherches qui furent à la fois novatrices et dominantes avant d'être largement remises en question, il est désormais important de sortir d'une sorte d'impasse intellectuelle. Sans doute le piège majeur a-t-il été l'ambition d'atteindre une macro-compréhension de la démographie, des familles et des sociétés historiques, à partir d'études de cas. Se pose et se repose alors sans cesse le problème de la représentativité des localités étudiées. Nous croyons qu'il est préférable de renverser radicalement la perspective et d'assumer la non-représentativité des monographies. La question essentielle est de bien saisir la spécificité d'un écotype, d'un écosystème, qui influence les familles et les individus, qui borne les options, les marges de manœuvre, qui façonne la " rationalité inconsciente " (Wrigley, 1978). Le deuxième temps de cet article s'attache à préciser 
les concepts environnementaux. Tous les auteurs que nous citons n'en sont pas nécessairement conscients, mais en fait, ils transforment le sens que l'on donne généralement à la monographie en la centrant non plus sur la population mais sur les familles (paragraphe 3 ) et les individus (paragraphe 4 ), considérés sous l'angle de leurs dynamiques de (sur) vie et de (re) production. À la description et à la compréhension de ces trajectoires, il convient de transposer le plaidoyer de Jacques Dupâquier (1981) qui, dans "Pour la démographie historique ", prônait le dépassement des moyennes et des agrégats statistiques au profit d'une analyse plus attentive de la dispersion autour des valeurs centrales. Ou, pour reprendre un mot d'Alfred Perrenoud, il ne faut pas négliger le rôle essentiel des minoritaires et/ou des comportements marginaux dans le fonctionnement des systèmes familiaux et sociaux.

\section{MODELES ECOLOGIQUES, SYSTEMES OUVERTS ET FORMES DE REGULATION}

En mettant en évidence le décalage entre les normes successorales et les pratiques de dévolution des biens (Bourdieu, 1987), les analyses historiques ont dépassé le déterminisme juridique qui marquait de nombreuses études sur les systèmes d'héritage, tout en renforçant les thèses écologiques qui ont focalisé leur attention sur les interdépendances entre les dynamiques démographiques, les institutions sociales et le milieu naturel dans les sociétés rurales préindustrielles. Par leur hétérogénéité culturelle et par leurs rigides contraintes environnementales, les espaces alpins ont représenté un terrain idéal pour tester le paradigme écologique (Cole, Wolf, 1974; Netting, 1981; Viazzo, 1989). Pour les historiens de la famille, le malthusianisme résidentiel de nombreuses communautés de montagne a été lu comme le résultat d'options familiales destinées à favoriser la succession unique et à limiter la croissance démographique en ajustant les possibilités d'établissement aux ressources disponibles (Burns, 1963; Burguière, 1986, 49; Barbagli, 1988, 545-547); une hypothèse qui, outre la validation fournie par diverses études (Ramella, 1984; Merzario, 1989)2 a suggéré la mise entre parenthèse des traits culturels - et donc des normes d'héritage - dans la construction des comportements démographiques (Berkner, Mendels, 1978).

Les critiques et les nuances formulées à l'égard des modèles écologiques sont bien connues (déterminisme environnemental, fermeture du modèle, pour une ample discussion (Viazzo, 1989)). Il est toutefois utile de rappeler les contributions de deux modèles alternatifs - le premier fondé sur le concept d'écotype (Gaunt, 1977) ${ }^{3}$ et le deuxième attribuant aux facteurs culturels un rôle actif dans la définition des comportements démographiques (Engelen, 1994) ${ }^{4}$ - qui ont proposé des approches plus articulées au problème des interrelations entre économie, écologie, démographie et pratiques successorales. L'impact du niveau d'intégration de l'économie dans le marché sur l'organisation domestique (Moring, 1999) ou sur la nuptialité (Ortmayr, 1995) illustre ce type de démarche qui, à plusieurs égards, complète celle parcourue par les historiens de la famille avec leurs enquêtes dédiées aux effets des pratiques successorales sur les normes d'accès au mariage. Considérons quelques exemples. Dans 
le Biellese (Italie du Nord), le célibat définitif relativement élevé semble s'expliquer par la norme juridique de la transmission indivise de la terre qui, en favorisant le maintien des fils dans la maison paternelle, finissait par en décourager plusieurs de se marier (Ramella, 1984). Dans un système lignager tel que celui de la FrancheComté, les pratiques de transmission et de gestion indivise des biens fonciers ont induit une nuptialité malthusienne tout à fait analogue à celle attestée dans les régions préciputaires des Pyrénées (Derouet, 1992; 1996). Le rôle de l'héritage émerge aussi dans les probabilités d'émigration. Dans la région allemande du Hesse-Cassel, les coutumes d'héritage en vigueur vers le milieu du $\mathrm{XIX}^{\mathrm{e}}$ siècle ont influencé de manière significative la structure économique des villages et, par ricochet, la tendance à l'émigration outre-mer (Wegge, 1999).

Ces résultats ont eu d'importantes conséquences sur le plan interprétatif. Ils ont notamment permis de reconsidérer les conséquences de la saturation de l'espace sur les modes de reproduction familiale. Le problème a donné lieu à de multiples analyses de la part des historiens nord-américains qui, à diverses reprises, ont illustré le parallélisme entre la saturation progressive du territoire et le passage à des formes de transmission plus sélectives (références dans Bouchard, 1996, 414-418). La similitude des processus de saturation est toutefois loin d'avoir donné lieu à des réponses uniformes. En opposant les " systèmes ouverts " (permettant le pluriétablissement) aux " systèmes clos " (ceux qui ne permettent qu'un seul établissement sur la terre), Bouchard (1996) a montré que dans le Saguenay (Québec), ce processus s'est soldé par la réorganisation des normes de la sociabilité familiale; la fin du pluriétablissement est allée de pair avec un égalitarisme étendu, accentué par les dispositions compensatoires en faveur des non-établis. Fortement explicatif pour le contexte québécois, ce modèle l'est probablement moins dans le cas des réalités européennes caractérisées par d'innombrables systèmes d'héritage. Par ailleurs, la définition de la saturation territoriale ne va pas sans poser de problèmes dans la mesure où elle peut recouvrir de multiples réalités démographiques et économiques. Ainsi, si en Franche-Comté l'ouverture économique de la fin du XIXe siècle a donné lieu à des pratiques successorales plus égalitaires, la terre perdant ses enjeux liés à la subsistance (Derouet, 1992), en Irlande, après la grande famine de 1846-1848 et l'émigration de masse qui suivit, on assista à l'émergence de formes d'héritage préciputaires dues au déséquilibre qui se créa cntrc la demande ct l'offrc d'hćriticrs (Kennedy, 1991).

Finalement, comme l'a suggéré Derouet (1994), il est probable que la typologie séparant les systèmes égalitaires des systèmes inégalitaires doive être remplacée par une autre typologie, distinguant les systèmes se tournant vers la restriction de la population et ceux qui optent pour l'expansion des ressources. Si les premiers ont adopté des réponses malthusiennes, afin de limiter le nombre d'héritiers (âge au mariage tardif, célibat définitif élevé, émigration), les seconds auraient répondu par des options expansives, centrées sur une gestion plus flexible de la terre et du travail. Bref, loin de donner lieu à des solutions uniformes, indépendantes des contextes géographiques et historiques, la saturation de l'espace a induit une 
multiplicité de réponses s'échelonnant, sur le plan démographique, du renforcement des mécanismes d'exclusion par le biais de la migration (Bonnain, 1986; Entchelecou, 1991; Lorenzetti, 1999), au durcissement des conditions d'accès au mariage (Head-König, 1992, 395), au blocage de la fécondité (FauveChamoux, 1987), à l'utilisation de l'illégitimité en tant que moyen d'exclusion (O'Neill, 1987). Sur le plan de la transmission, en revanche, les solutions, parfois mises en œuvre de manière séquentielle ou complémentaire (Siddle, 1986; Fontaine, 1995), se sont étalées de l'apparition de formes de transmission inégalitaires (Derouet, 1992) à l'élargissement des enjeux autour des stratégies de compensation en faveur des non-établis et à la restructuration de la sociabilité familiale (Bouchard, 1996), jusqu'aux ajustements s'opérant sur le marché foncier (Segalen, 1985; Sabean, 1990).

Ces résultats, qui confirment la multiplicité des aspects contribuant à la définition des stratégies successorales, corroborent en même temps l'importance des facteurs culturels et des traditions juridiques. Une importance que les études sur les relations entre la reproduction familiale et les alliances matrimoniales ont amplement mis en évidence. En Italie méridionale, par exemple, la cohérence des divers systèmes agraires, démographiques et familiaux était fondée sur un ensemble de valeurs bâties autour des règles de formation et de perpétuation des lignages, des coutumes de transmission des patrimoines, des pratiques dotales et des formes de réciprocité des échanges (Delille, 1985); un résultat saisissant, qui renvoie au problème des configurations parentales impliquées dans l'usage de la terre (Salitot, 1988) ainsi qu'à celui de la signification des stratégies matrimoniales et d'alliance familiale. En effet, si dans les systèmes égalitaires elles étaient souvent utilisées dans le but de favoriser la recomposition des patrimoines familiaux grâce aux jeux de la consanguinité et des renchaînements d'alliance (Segalen, 1985; Sabean, 1990), dans d'autres cas, leur signification mettait en jeu les mécanismes de la réciprocité interfamiliale (Merzario, 1981) ou des logiques de perpétuation lignagère (Lenclud, 1991) qui allaient au-delà des considérations strictement économiques et qui suggèrent le rôle de la parenté en tant que support de l'ordre et de l'organisation sociale et moteur des dynamiques de formation de la stratification sociale (Sabean, 1998).

\section{REPRODUCTION FAMILIALE ET REPRODUCTION DEMOGRAPHIQUE}

La complexité du panorama des systèmes familiaux et des solutions mises en œuvre afin de répondre aux problèmes de l'ajustement entre les ressources et les bouches à nourrir, a favorisé l'élargissement des approches aux pratiques de la transmission et de la reproduction familiale. Elle a notamment conduit les historiens à modifier l'optique d'analyse qui, grâce à la mise au point de nouvelles méthodes (il suffit de penser à la rencontre des méthodes nominatives de la démographie historique et de l'analyse des corpus d'actes notariés) ont de plus en plus valorisé les perspectives microhistoriques.

Par ce glissement d'optique, les recherches ont progressivement focalisé leur intérêt sur la famille en tant que système social, intégrant, outre les fonc- tions de production et de reproduction, 
celles de transmission et de distribution. La reproduction familiale, à savoir l'ensemble des dispositions à travers lesquelles chaque génération dispose de ses avoirs en faveur de la génération suivante, coagule donc les multiples enjeux et les stratégies à travers lesquelles se négocient des objectifs souvent contradictoires, comme la nécessité d'assurer l'intégrité et la viabilité des exploitations et des patrimoines familiaux, la volonté d'établir le maximum d'enfants sur la terre et le souhait de sécurité des parents durant leur vieillesse.

Le bilan d'une vingtaine d'années de recherches sur les stratégies de la reproduction familiale est sans doute très riche. Il reste toutefois à évaluer l'étonnante diversité des solutions disponibles ou " inventées " par les familles dans leur effort reproductif. Deux possibilités - à notre avis complémentaires - pourraient éviter l'impasse intellectuelle que semble entraîner la fragmentation des expériences et des stratégies mises en évidence par la recherche historique. La première consiste à réélaborer la notion de stratégie: en terme de régularités et de cohérence des comportements face au problème de la reproduction familiale (Derouet, 1994) ou bien par rapport à une série de paramètres (la structure des patrimoines, les règles $\mathrm{du}$ choix du conjoint, l'âge tendanciel au mariage, le critère de dotation des femmes, les règles d'héritage, etc.) de manière à devenir une catégorie analytique forte (Casanova, 1997). La deuxième possibilité, en revanche, est de valoriser les marges et la diversité, de manière à saisir la multiplicité des solutions à disposition des groupes domestiques.

Dans les deux cas, l'analyse démographique longitudinale et celle sur le renouvellement des populations peuvent offrir de précieuses indications. M. Segalen (1985) l'avait d'ailleurs souligné il y a quelques années, en affirmant l'impossibilité de saisir les pratiques de la transmission sans savoir combien d'enfants survivent à chaque génération. En exploitant les données de l'enquête TRA, M. Dürr (1992) avait montré que la reproduction démographique traduisait une mobilité et un accès au mariage variant en fonction de multiples paramètres démographiques et socio-économiques. Les enquêtes suivantes ont ultérieurement approfondi cette piste qui a révélé l'effet d'entonnoir par lequel une minorité des couples d'une cohorte de mariages participait en mesure prépondérante à la constitution de la génération suivante (Brunet et al., 1998 , 4). Les études sur la reproduction démographique différentielle ont d'ailleurs insisté sur les mécanismes de l'exclusion définis par le croisement des stratégies de gestion du célibat définitif et de l'émigration d'une part, des effets du sexe et du rang de naissance d'autre part (Perrenoud, 1993; Bideau, Brunet, 1996; Bouchard, 1996). Si l'on considère les stratégies d'émigration, dans le Pays de Herve entre 1846 et 1900 , le rang de naissance n'impliquait aucun effet sur les probabilités de quitter la commune, seul le calendrier des départs dénotant une différence entre les membres des fratries (Neven, 2000a). Dans les communautés tessinoises au XIX $X^{e}$ siècle, en revanche, l'émigration définitive était corrélée au rang de naissance (et au sexe) des individus, les cadets ayant plus de probabilités de quitter définitivement le village que leurs frères aînés (Lorenzetti, 1999, 278290). Des tendances contrastées s'esquissent aussi à l'égard de la nuptialité. L'étude de Brennan, James et Morrill 
(1982) attribuait au système d'héritage un rôle décisif, la transmission inégalitaire - contrairement à celle égalitaire donnant lieu à une relation significative entre le rang de la naissance d'une part, les opportunités et le rang du mariage d'autre part. Ce résultat a été confirmé par diverses enquêtes sur des systèmes à héritage sélectif ou préciputaire (Bauer, 1983; Bonnain, 1986; Guinnane, 1992a; Lorenzetti, 1999) mais, encore une fois elles doivent être nuancées par les exceptions révélées par la recherche. A Esparros, par exemple, l'imposition de l'égalité entre les héritiers par le Code Napoléon a ouvert de plus larges espaces pour le mariage des cadets qui, contrairement à leurs frères aînés, ont brisé le frein malthusien (Fauve-Chamoux, 1995)

La distinction entre les aînés et les cadets perd naturellement une large partie de sa pertinence dans les systèmes égalitaires. Au Saguenay (Québec) par exemple, aucune règle préférentielle ne distinguait les héritiers qui bénéficiaient de la donation parentale, se mariaient et s'établissaient en fonction de leur rang de naissance (Bouchard, 1996, 210). Des exceptions indiquent toutefois que l'égalité devant l'héritage pouvait s'accommoder de discriminations face au mariage. Dans la communauté égalitaire valaisanne étudiée par Netting (1981), bien que le rang de naissance n'eût aucun impact sur les probabilités de mariage, les cadets tendaient à se marier plus tard que leurs frères aînés en raison des nécessités familiales qui incitaient les parents à les retenir à la maison pour qu'ils puissent les aider durant leur vieillesse.

Au-delà de ces remarques, les analyses démographiques offrent d'importantes indications concernant l'intensité du renouvellement démographique et les composantes démographiques de l'exclusion au sein des familles. Des indications qui, insérées dans une grille analytique associant d'autres paramètres (systèmes familiaux de transmission ou de succession, inégalités socio-économiques, structure de la propriété foncière, rôle des alliances matrimoniales, influence de l'environnement urbain, contexte culturel) permettent de comparer les différents systèmes de reproduction familiale (Bouchard, Goy, 1992, 423-424).

Très féconde de résultats, cette optique a d'ailleurs permis de regarder la reproduction familiale non pas seulement comme l'instrument à travers lequel on assurait la transmission des biens, mais aussi comme le moyen par lequel se structuraient les relations interpersonnelles (Goody, 1976). Les études sur les implications relationnelles intrafamiliales de la transmission (Medick, Sabean, 1984), celles sur les cadets (Segalen, Ravis-Giordani, 1994) ou celles sur le destin des exclus de l'héritage (Bouchard G., Dickinson J. A., Goy J., 1998) illustrent de manière concrète cette orientation qui, en outre, offre d'importantes perspectives concernant l'organisation et le fonctionnement des systèmes de parenté saisis, selon les cas, dans leur dimension verticale (Delille, 1985) ou horizontale (Levi, 1985) et dans leurs implications sur les modes de la reproduction familiale.

\section{ARTICULER COURS DE LA VIE ET DYNAMIQUES FAMIIIALES}

Les études centrées sur le cours de la vie placent l'individu au cour de l'analyse. Elles ne font pas, pour autant, abstraction de son environnement. Au contraire, et par définition, une approche du cours de 
la vie "includes both micro- and macrolevel influences on individual behavior " (Dykstra, Van Wissen, 1999, 5). L'homme et les diverses étapes qu'il traverse au cours de son existence est ainsi replacé dans la dynamique de son ménage, mais aussi, autant que possible, dans son environnement socio-économique au sens large (famille étendue, économie régionale, circonstances démographiques locales, etc.).

Du point de vue de la transmission, les études sur la reproduction familiale ont longtemps concentré leur regard sur deux circonstances centrales, à savoir le mariage - acte d'investiture du successeur dans de nombreux systèmes inégalitaires - et le décès - le moment marquant le passage des biens d'une génération à l'autre, surtout dans les systèmes égalitaires où prévaut la pratique testamentaire. En distinguant la transmission des biens et celle de l'autorité ou du pouvoir (Augustins, 1989), diverses enquêtes ont toutefois apporté d'importantes nuances à ce schéma; il suffit de penser aux différences existant entre la famille-souche pyrénéenne (Fauve-Chamoux, 1984; 1987) où le fils héritier-successeur n'obtenait la propriété des biens de la maison que lors du décès du père, et celle attestée en Autriche (Berkner, 1976; Sieder, Mitterauer, 1983) où le retrait des vieux parents promouvait le fils successeur en tant que nouveau propriétaire. Même l'option en faveur de la primogéniture ou de l'ultimogéniture n'était pas sans conséquences, dans la mesure où elle pouvait influencer les scansions marquant le développement des ménages. En ce sens, c'est grâce au couplage des informations démographiques et des séries notariales que l'on a pu saisir la complexité extrême de la transmission qui, loin d'être un acte isolé, était parfois un processus s'étendant tout au long du cours de vie individuel et familial et ponctué par des moments particuliers, coïncidant avec des phases de rupture ou de restructuration du ménage. La reconstitution des séries notariales révèle ici tout son intérêt dans la mesure où elle permet de replacer chaque acte (transactions, donations, contrats dotaux, testaments, etc.) à l'intérieur de la trajectoire familiale et individuelle et de reconstituer les logiques et les stratégies familiales concernant la gestion des ressources.

En reprenant les réflexions du modèle de Chayanov, les premières études dans cette direction ont illustré les relations entre le cours de vie familial et la composition des patrimoines (Levi, 1976; Derouet, 1982). Le modèle de Chayanov repose sur l'observation de l'évolution du niveau de vie des familles paysannes russes en fonction du cycle de vie familiale. Les ménages rencontraient une première période de crise lorsque le nombre des enfants s'accroissait et que ceux-ci étaient encore trop jeunes pour travailler (Thorner et al., 1966). Berkner avance l'hypothèse d'un rôle prépondérant des domestiques lors de cette phase difficile, ceux-ci étant censés suppléer au manque de force de travail (Berkner, 1972, 415). Cette interprétation a été confirmée par les recherches menées par Lundh sur la Scanie suédoise au XIXe siècle. Particulièrement " in tenants households, servants were, as Berkner suggests, more important to the labour force during the first phase of the family's life cycle. When the children grew up, the relative importance of servants decreased $"$ (Lundh, 1995, 61). Dans un contexte socio-économique totalement différent, celui d'une petite ville industrielle belge au XIX siècle 
(Tilleur), pour passer la première période difficile, les ménages ouvriers privilégiaient une intensification du labeur de l'homme adulte, du principal wage earner en somme, ainsi qu'une mise au travail aussi précoce que possible des enfants aînés. Si elle était efficace sur le moment, cette solution n'était toutefois pas sans conséquence, à terme, sur le cours de la vie individuelle, dans la mesure où les hommes - principalement les mineurs payaient par une sénescence précoce et une surmortalité nette dans la vieillesse, le prix des efforts fournis dans l'adolescence et à l'âge adulte (Neven, 2000b).

La deuxième phase difficile survient pour le ménage lorsque les enfants grandissent et quittent peu à peu le foyer, laissant seuls leurs parents vieillissants (Lundh, 1995, 61). Cette piste, réinterprétée par M. Segalen (1985) dans le cas des systèmes égalitaires, a été récemment reprise par des études qui se sont penchées sur les phases des investissements et des désinvestissements fonciers le long du cours de vie individuel. Chez les vignerons de Chartres (Béaur, 1991), comme chez les ouvriers protoindustriels d'une communauté zurichoise (Pfister, 1994) ou les petits propriétaires de quelques villages d'émigrants tessinois (Lorenzetti, 1999), les achats et les ventes foncières se déroulaient selon une séquence liée aux capacités productives de l'individu ou du ménage et à la nécessité de disposer de réserves pour la vieillesse, lorsque l'incapacité de travailler et la diminution du revenu familial imposaient la vente des propriétés dont on ne pouvait plus assurer l'exploitation.

Si les résultats de telles analyses révèlent d'intéressantes convergences entre les divers contextes observés, il reste à comprendre dans quelle mesure la scansion des naissances et la configuration familiale pouvaient affecter l'investissement (ou le désinvestissement) foncier. Néanmoins, un acquis essentiel est la mise en évidence du rôle joué par les échanges de terres pour sécuriser la fin de vie des personnes âgées dans les sociétés de propriétaires. Dès le XVII siècle, les gros fermiers de l'île-de-France - propriétaires cultivateurs ou directeurs de grandes exploitations - avaient recours à des méthodes d'ultimogéniture pour se garantir une vieillesse sans soucis par le biais des contrats de mariage, généralement ceux des cadets: les parents accordaient une dot moyennant le logement du parent survivant dans la ferme cédée, ainsi que la jouissance de quelques terres, souvent réduites au potager et à la basse-cour (Moriceau, 1985, 129). Par ailleurs, « la règle du groupe interdisait tout partage en dehors de la volonté du survivant " (Idem, 35). En suivant l'histoire d'une famille de grands fermiers parisiens du $\mathrm{XVII}^{\mathrm{e}}$ au XXe siècle, Moriceau et PostelVinay $(1992,165)$ confirment, dans un régime de partage égalitaire, la volonté de préserver le siège d'exploitation, par " une sorte de droit de préemption en faveur du successeur de la ferme " et l'existence d'ententes entre les héritiers.

Même dans des sociétés de locataires peu aisés, sans qu'il y ait d'ailleurs héritage à la clé, plusieurs études ont mis en évidence, dans l'est de la Belgique, des systèmes de prise en charge des personnes âgées par leurs enfants, les cadets de préférence (Alter, 1996, 16-17; Alter, Cliggett, Urbiel, 1996; Alter, Oris, 1999; Neven 2000a, 546). Ces recherches relisent le mariage tardif en mettant l'accent sur le célibat définitif et sur le rôle spécifique, disproportionné, tenu par les enfants célibataires dans les soins aux personnes âgées. Dans le Pays de 
Herve par exemple, entre 1846 et 1900 , plus de $80 \%$ des enfants qui cohabitaient avec un géniteur âgé de 55 ans ou plus étaient célibataires (Neven, 2000a, 571). Le régime démographique en vigueur dans la région (mariage tardif, pas de fécondité d'arrêt) permettait à la plupart des "vieux " d'échapper au empty nest, à condition toutefois qu'ils aient eu des enfants (Alter, 1996; Alter, 1999; Neven, 2000a; Alter, Neven, Oris 2000). Parmi ces derniers, un groupe minoritaire de vieux garçons et de vieilles filles, socialement et en tout cas démographiquement marginal, avait une double fonction essentielle en réduisant la pression démographique et, à l'intérieur du système familial, en évitant aux plus âgés d'être confrontés à la dureté potentielle de la famille nucléaire.

\section{CONCLUSION}

Dans un bilan tracé il y a dix ans, M. King (1990) faisait état des multiples barrières s'interposant dans le dialogue entre la démographie historique et l'histoire de la famille. Barrières dues aux différences dans la perception de la famille en tant qu'objet d'étude et à l'inadéquation des méthodes de la démographie par rapport aux questions soulevées par les historiens de la famille. Bien que n'ayant pas empêché de nouer des liens étroits, la différence entre les deux disciplines s'est traduite par des démarches pour certains aspects complémentaires, les historiens démographes s'intéressant prioritairement à l'influence des processus démographiques sur les formes et les dynamiques des groupes domestiques et les historiens de la famille s'efforçant d'illustrer l'impact des systèmes familiaux sur les comportements démographiques (Skinner, 1999).
Au-delà de ces écarts, les deux approches ont montré que l'analyse individuelle ne fait fi ni des dynamiques du groupe domestique, ni de l'environnement familial, qui sont au contraire des éléments déterminants pour analyser et comprendre les parcours de vie. Il est trop facile de lire cette démarche comme un anachronisme de fin de $\mathrm{XX}^{\mathrm{c}}$ siècle, un simple reflet des valeurs réputées individualistes de la société contemporaine. Il s'agit bel et bien d'un renouveau méthodologique qui s'appuie de plus en plus sur un nouvel outillage statistique, l'event history analysis, ou analyse des biographies (voir Bocquier, 1996 pour une présentation accessible; Gutman, Alter, 1993 et Alter, 1998 pour les applications historiques). L'orientation est fondamentalement micro-démographique puisque l'individu est l'unité statistique de base. Initialement développée dans le milieu médical, cette méthode met l'accent, en démographie historique, sur l'approche multivariée des transitions individuelles pensées dans un contexte de plus en plus ouvert. Kertzer et ses collègues (Hogan, Kertzer, 1985; Kertzer, Hogan, 1989) ont été les premiers à traiter les réseaux familiaux au-delà des ménages. Bengtsson (1993) a inclus dans les modèles d'analyses des séries temporelles de prix, de salaires ou de températures qui permettent de mesurer l'impact des conjonctures, voire des structures socioéconomiques locales ou régionales, sur les parcours de vie. Depuis quelques années, le Projet EurAsien pour une Histoire Comparée de la Population et de la Famille intègre et développe ces apports dans une démarche interdisciplinaire novatrice (Bengtsson et al., 1998).

Des perspectives intéressantes s'ouvrent aussi dans le domaine de la reproduction familiale. La séquence et 
l'enchaînement des transactions immobilières et des opérations liées à la dévolution des biens d'une génération à l'autre, l'écart temporel entre le décès des parents et le partage de l'héritage, ou entre le mariage et le versement dotal, ne sont que quelques pistes qui, grâce au couplage des informations démographiques, notariales, cadastrales etc., devraient permettre de replacer le cours de la vie individuel dans son contexte familial, social et économique. En outre, dans le cas de sociétés qui vivaient massivement dans l'incertitude du lendemain, les méthodes nouvelles devraient permettre de mieux évaluer l'impact du court terme, de l'accidentel (décès subit du père, crise épidémique ou céréalière, etc.) sur les stratégies de long terme à l'échelle familiale, c'est-à-dire celles de la transmission entre générations.

Luigi LORENZETTI

Université de Genève Département d'Histoire Économique 102 bd. Carl-Vögt, CH-1211Genève Suisse Luigi.Lorenzetti@histec.unige.ch Muriel Neven Fonds National de la Recherche Scientifique et Université de Liège Laboratoire de Démographie, 16 quai Roosevelt $B-4000$ Liège Belgique Muriel.Neven@ulg.ac.be

\section{NoTES}

1. " Individualisme, déchristianisation, vie urbaine et société "éclairée " servent de toile de fond à la mise en place d'un modèle familial urbain qui se caractérise à la fois par un certain respect de la femme et de l'enfant, donc par une fécondité limitée, et le rejet des personnes âgées. Mais ce modèle bourgcois n'est pas facilement transposable et l'on voit les sociétés paysannes s'adapter par d'autres voies à la modernité " (p. 366).

2. Il faut néanmoins citer les exceptions soulignées par Netting (1981) et Viazzo (1989).

3. Selon Gaunt, les comportements démographiques et l'organisation domestique des sociétés rurales préindustrielles ne dépendaient pas uniquement des contraintes écologiques mais étaient plutôt le résultat d'un ensemble d'influences et de causalités hiérarchiques définies par l'écotype, à savoir par la configuration des formes d'exploitations des ressources à l'intérieur du système économique local (Rudolph, 1995; Mitterauer, 1995, 28-36).

4. D'après Engelen, les facteurs culturels peuvent avoir une marge d'autonomie élevée par rapport aux conditionnements économiques, ce qui signifie que les mécanismes et les comportements démographiques peuvent dépendre entièrement des valeurs sociales, des traditions et des coutumes partagées par la population.

5. Par une perspective méthodologique complémentaire (analyse agrégée) P. A. Rosental (1991) a montré que l'introduction des normes égalitaires en France n'a induit aucun effet direct sur les comportements féconds de la population. 


\section{RÉFÉRENCES BIBLIOGRAPHIQUES}

ALTER, G. (1996), “The European Marriage Pattern as Solution and Problem: Households of the Elderly in Verviers, Belgium, 1831", 3-19, in Les systèmes démographiques du passé, A. Bideau, A. Perrenoud, K.A. Lynch., G. Brunet G. (éd.), Lyon, Programme Rhône-Alpes Recherches Sciences Humaines.

ALTER, G. (1998), "L'Event History Analysis en démographie historique. Difficultés et perspectives ", Annales de démographie bistorique, 2, 25-35.

ALTER, G. (1999), "Vieillir dans les ménages d'une ville industrielle: l'impact de l'âge de migration (Verviers, début du XIX siècle) ", Annales de démographie historique, 2, 9-29.

Alter, G., Oris, M. (1999), "Access to Marriage in the East Ardennes during the XIX ${ }^{\text {th }}$ Century", 133-151, in Marriage and Rural Economy. Western Europe since 1400, I. Devos, L. Kennedy (eds), Tielt Brepols.

Alter, G., Cliggett, L., Urbiel, A. (1996), "Household Patterns of the Elderly and the Proximity of Children in a Nineteenth-Century City, Verviers, Belgium, 1831-1846", 30-52, in Aging and Generational Relations over the Life Course. A Historical and Cross-Cultural Perspective, T. K. Hareven (ed.), Berlin, New York, De Gruyter.

Alter, G., NeVEN, M., ORIS, M. (2000, à paraître), "How Hard Was the Road to Modernization! Mortality in XIX ${ }^{\text {th }}$ Century Eastern Belgium", in Families, the Economy, and Death in Past Times: Relationships between Economic Conditions, Household Context, and Mortality in Europe and Asia before the $X X^{t}$ ' Century, T. Bengtsson, C. Campbell (eds).

Augustins, G. (1989), Comment se perpétuer? Devenir des lignées et destins des patrimoines dans les paysanneries européennes, Nanterre, Société d'ethnologie.
BARBAGLI, M. (1988), Sotto lo stesso tetto. Mutamenti della famiglia in Italia dal XV al XIX secolo, Bologna, Il Mulino.

BAUER, R. (1983), Family and Property in a Spanish Galician Community, Stanford (thèse non publiée).

BÉAUR, G. (1991), "Investissement foncier, épargne et cycle de vie dans le pays chartrain au XVIII" siècle ", Histoire \& Mesure, VI, 3-4, 275-288.

Bengtsson, T. (1993), "Combined TimeSeries and Life-Event Analysis: the Impact of Economic Fluctuations and Air Temperature on Adult Mortality by Sex and Occupation in a Swedish Mining Parish, 1757-1850", 239-256, in Old and New Methods in Historical Demography, D. Reher, R. Schofield (eds), Oxford, Clarendon Press.

BERKNER, L. K. (1972), “The Stem Family and the Developmental Cycle of the Peasant Household: an EighteenthCentury Austrian Example", American Historical Review, 77 (2), 398-418.

BERKNER, L. K. (1975), "The Use and Misuse of Census Data for the Historical Analysis of Family Structure", Journal of Interdisciplinary History, 4, 721-738.

BERKNER, L. K. (1976), "Inheritance, Land Tenure and Peasant Family Structure: a German Regional Comparison", 71-95, in Family and Inheritance. Rural Society in Western Europe, 1200-1800, J. Goody, J. Thirsk, E. P. Thompson (eds), Cambridge, Cambridge University Press.

Berkner, L. K., Mendels, F. F. (1978), "Inheritance System, Family Structure, and Demographic Patterns in Western Europe, 1700-1900", 209-223, in Historical Studies of Changing Fertility, C. Tilly (ed.), Princeton, Princeton University Press.

Bideau, A., Brunet, G. (1996), "Rester ou partir: choix individuel et logique familiale ", 69-82, in Les systèmes 
démographiques du passé, A. Bideau, A. Perrenoud, K.A. Lynch, G. Brunet (éd.), Lyon, Programme Rhône-Alpes Recherches Sciences Humaines.

BOCQUIER, P. (1996), L'analyse des enquêtes biographiques à l'aide du logiciel STATA, Paris, Documents \& Manuels CEPED.

BonnaIN, R. (1986), "Nuptialité, fécondité et pression démographique dans les Pyrénées 1769-1836 ", 87-110, in Les Baronnies des Pyrénées. Anthropologie et histoire, permanences et changements. Tome II. Maison, espace, famille, I. Chiva, J. Goy (éd.), Paris, EHESS.

BOUCHARD, G. (1996), Quelques arpents d'Amérique. Population, économie, famille au Saguenay 1838-1971, Montréal, Éditions Boréal.

Bouchard, G., Dickinson, J. A., Goy J. (sous la dir. de) (1998), Les exclus de la terre en France et au Québec XVIT-XX siècles. La reproduction familiale dans la différence, Sillery (Québec), Les Éditions du Septentrion.

BOUChard, G., GoY, J. (1992), "Conclusion ", 423-429, in Transmettre, hériter, succéder. La reproduction familiale en milieu rural. France Québec XVIIT-XIX siècles, R. Bonnain, G. Bouchard, J. Goy (éd.), Lyon, Presses universitaires de Lyon.

Bourdieu, P. (1987), " De la règle aux stratégies ", Choses dites, Paris, Éd. de Minuit, 75-94.

BRENNAN, E. R., JAMES, A. V., MORRILl, W. T. (1992), "Inheritance, Demographic Structure, and Marriage: a Cross-Cultural Perspective", Journal of Family History, 7, 289-298.

Brunet, G., Fauve-Chamoux, A., Oris, M. (1998), "Le choix du conjoint: méthodes multiples, dialogue difficile ", 3-12, in Le choix du conjoint, G. Brunet, A. Fauve-Chamoux, M. Oris (éd.), Lyon, Programme Rhône-Alpes Recherches Sciences Humaines.

BURGUIĖRE, A. (1986), "Les cent et une familles de l'Europe ", 17-91, in Histoire de la famille, vol. 2. Le choc des modernités,
A. Burguière, Ch. Klapish-Zuber, M. Segalen, F. Zonabend (éd.), Paris, Armand Colin.

BURguiére, A. (1995), "L'anthropologie historique ", 171-185, in L'histoire et le métier d'historien en France 1945-1995, F. Bédarida (éd.), Paris, Éditions de la Maison des Sciences de l'Homme.

BURNS, R. K. (1963), "The Circum-Alpine Area: A Preliminary View", Anthropological Quarterly, 30, 130-155.

CASANOVA, C. (1997), La famiglia italiana in età moderna: ricerche e modelli, Roma, La Nuova Italia Scientifica.

Chartier, R. (1992), "La ligne SaintMalo-Genève ", 739-775, in Les lieux de mémoire, t. III. Les France. Conflits et partages, P. Nora (éd.), Paris, Gallimard.

Cole, J. W., WOLF, E. R. (1974), The Hidden Frontier: Ecology and Ethnicity in an Alpine Valley, New York, San Francisco, Academic Press.

Delille, G. (1985), Famille et propriété dans le Royaume de Naples (XV-XIX siècles), RomeParis, École française de Rome.

Derouet, B. (1982), "Famille, ménage paysan et mobilité de la terre et des personnes en Thimerais au XVIII' siècle ", Etudes rurales, 86, avril-juin, 47-56.

DeRoueT, B. (1992), "La succession et l'héritage masculin en Franche-Comté: histoire et logique d'une mutation ", 243-263, in Transmettre, hériter, succéder. La reproduction familiale en milieu rural, FranceQuébec XVIIr-XX' siècles, R. Bonnain, G. Bouchard, J. Goy (éd.), Lyon, Presses universitaires de Lyon.

Derouet, B. (1994), "Transmettre la terre. Origines et inflexions récentes d'une problématique de la différence ", Histoire et sociétés rurales, 2, 33-67.

DEROUET, B. (1996), "Nuptialité et reproduction familiale dans les systèmes d'héritage masculin: Réflexions à partir de l'exemple de la Franche Comté (XvIrcXVIII siècles) ", 41-66, in Les systèmes 
démographiques du passé, A. Bideau, A. Perrenoud, K. A Lynch, G. Brunet (éd.), Lyon, Programme Rhône-Alpes Recherches Sciences Humaines.

Derouet, B. (1998), "Pratiques de l'alliance en milieu de communautés familiales (Bourbonnais, XVIIrc siècle) ", 227-251, in Le choix du conjoint, G. Brunet, A. Fauve-Chamoux., M Oris (éd.), Lyon, Programme Rhône-Alpes Recherches Sciences Humaines.

DEVOLDER, D. (2000, à paraître), "Effects of the European Late Marriage Pattern on Kinship. A Study Using a Microsimulation Model", in When Dad Died. Individuals and Families Coping with Family Stress, R. Derosas, O. Saito, M. Oris M. (eds).

DUPÂQUIER, J. (1981), Pour la démographie historique, Paris, Presses Universitaires de France.

DÜRR, M. (1992), "Les uns croissent et multiplient, les autres non. La reproduction différentielle des familles ", 439-459, in La société française au XIX siècle. Tradition, transition, transformations, J. Dupâquier, D. Kessler (éd.), Paris, Fayard.

DykstRa, P.A., VAN Wyssen, L. (1999), "Introduction: the Life Course Approach as an Interdisciplinary Framework for Population Studies", 1-22, in Population Issues. An Interdisciplinary Focus, P. A. Dykstra, L. Van Wyssen (eds), New York, Kluwer Academic/Plenum Publisher.

Engelen, T. (1994), "Family, Production and Reproduction: on the Relationship between Economic and Demographic Process", Economic and Social History in the Netherlands, 6, 61-82.

ETCHELECOU, A. (1991), Transition démographique et système coutumier dans les Pyrénées occidentales, Paris, INED.

FAUVE-ChamouX, A. (1984), "Les structures familiales au royaume des famillessouches: Esparros ", Annales E.S.C., 3, 513-528.

Fauve-Chamoux, A. (1987), "Le fonctionnement de la famille-souche dans les
Baronnies des Pyrénées avant 1914 ", Annales de démographie historique, 241-262.

Fauve-Chamoux, A. (1995), "The Stem Family, Demography and Inheritance: the Social Frontiers of Auto-Regulation", 86113, in The European Peasant Family and Society. Historical Studies, R. Rudolph (ed.), Liverpool, Liverpool Univ. Press.

Fauve-Chamoux, A., Wall, R. (1997), "Nuptialité et famille ", 344-368, in Histoire des populations de l'Europe. I. Des origines aux prémices de la révolution démographique, J.-P. Bardet, J. Dupâquier (éd.), Paris, Fayard.

Fauve-Chamoux, A., Ochiai, E. (1998) (sous la dir. de), Maisons et famille-souche: perspectives eurasiennes, Proceedings of the C18 Session Twelfth International Economic History Congress, août 1998, Kyoto, International Research Center for Japanese Studies, 468 p.

FonTAINE, L. (1995), “Devoluzione dei beni nelle valli alpine del Delfinato (XVII-XVIII secolo)", Quaderni Storici, 1, 135-154.

GaUNT, D. (1977), "Pre-Industrial Economy and Population Structure", Scandinavian Journal of History, 3, 2, 183-210.

Goodx, J. (1976), "Introduction", 1-9, in Family and Inheritance. Rural Society in Western Europe, 1200-1800, J. Goody, J. Thirsk, E.P. Thompson (eds), Cambridge, Cambridge University Press.

GuINNANE, T. (1992a), "Intergenerational Transfers, Emigration and the Rural Irish Household System", Explorations in Economic History, 29, 456-476.

GuINNANE, T. (1992b), "Age at Leaving Home in Rural Ireland, 1901-1911", Journal of Economic History, 52, 651-674.

Gutmann, M., Alter, G. (1993), "Family Reconstitution as Event History Analysis", 159-177, in Old and New Methods in Historical Demography, D. Reher, R. Schofield (eds), Oxford, Clarendon Press.

Hajnal, J. (1983), "Two Kinds of Preindustrial Household Formation System", 
Population and Development Review, 3, 449-495.

HEAD-KÖNIG, A.-L. (1992), "La reproduction familiale et sociale dans les campagnes helvétiques (XVIII ${ }^{\mathrm{c}}$-XIXe siècles) ", 387-399, in Transmettre, hériter, succéder. La reproduction familiale en milieu rural. France Québec XVIIT'-XIX siècles, R. Bonnain, G. Bouchard, J. Goy (éd.), Lyon, Presses Universitaire de Lyon.

HogaN, D., KerTZER, D. (1985), "Longitudinal Approaches to Migration in Social History", Historical Methods, 18 (1), 20-30.

JANSSENS, A. (1993), Family and Social Change. The Household as a Process in an Industrializing Community, Cambridge, Cambridge University Press.

KenNEDY, L. (1991), "Farm Succession in Modern Ireland: Elements of a Theory of Inheritance ", Economic History Review, 3, 477-499.

Kertzer, D., Hogan, D. (1989), Family, Political Economy and Demographic Change, Madison, University of Wisconsin Press.

KING, M. (1990), "All in the Family? The Incompatibility and Reconciliation of Family Demography and Family History", Historical Methods, 1, 32-40.

LASLETT, P. (1972), "Introduction: The History of the Family", 1-89, in Household and Family in Past Time, P. Laslett, $\mathrm{R}$. Wall (eds), Cambridge, Cambridge University Press.

LASLETT, P. (1983), "Family and Household as Work Group and Kin Group: Areas of Traditional Europe Compared", 513-563, in Family Form in Historic Europe, R. Wall, J. Robin, P. Laslett (eds), Cambridge, Cambridge University Press.

Le Roy Ladurie, E. (1972), "Structures familiales et coutumes d'héritage ", Annales E.S.C., 27, 825-846.

LENCLUD, G. (1991), " Mariage, transmission des biens et reproduction sociale dans la Corse traditionnelle ", 147-176, in Les complexités de l'alliance, vol. II, Les systemes complexes d'alliance matrimoniale, F. Héritier-
Augé, E. Copet-Rougier, (textes rassemblés et présentés par), Paris, Éditions Archives Contemporaines.

LEVI, G. (1976), “Terra e strutture familiari in una comunità piemontese del 700", Quaderni Storici, sett.-dic., 1095-1114.

LEVI, G. (1985), L'eredità immateriale: carriera di un esorcista nel Piemonte del Seicento, Torino, Einaudi.

LORENZETTI, L. (1999), Economie et migration au XIX siècle. Les stratégies de la reproduction familiale au Tessin, Berne [et al.], Peter Lang.

LUNDH, C. (1995), "Households and Families in Pre-Industrial Sweden", Continuity and Change, 10 (1), 33-68.

Medick, H., Sabean, D. W. (eds.) (1984), Interest and Emotion. Essays on the Study of Family and Kinship, Cambridge, Cambridge University Press/Paris, Maison des Sciences de l'Homme.

Merzario, R. (1981), Il paese stretto. Strategie matrimoniali nella diocesi di Como, secoli XVI-XVIII, Torino, Einaudi.

MERZARIO, R. (1989), Il capitalismo nelle montagne. Strategie familiari nelle prime fasi di industrializzazione nel comasco, Bologna, Il Mulino.

Mitterauer, M. (1995), "Peasant and Non-Peasant Family Forms in Relation to the Physical Environment and the Local Economy", 26-48, in The European Peasant Family and Society. Historical Studies, R. Rudolph R. (ed.), Liverpool, Liverpool University Press.

MoriceaU, J.-M. (1985), " Un système de protection sociale efficace: l'exemple des vieux fermiers de l'île-de-France (XVII ${ }^{\text {e- }}$ début XIXe siècle) ", Annales de démographie historique, 127-144.

Moriceau, J.-M., Postel-VINaY, G. (1992), Ferme, entreprise, famille. Grande exploitation et changements agricoles, $X V I I^{2}-X I X^{2}$ siècles: les chartiers, XVII -XIX siècles, Paris, Éditions de l'École des Hautes Études en Sciences Sociales. 
MORING, B. (1999), "Economic and Ecologic Determinants of Household Organisation in the Northeastern Baltic Region", Scandinavian Economic History Review, 47 (3), 48-68.

NetTing, R. (1981), Balancing on an Alp. Ecological Change and Continuity in a Swiss Mountain Community, Cambridge, Cambridge University Press.

Neven, M. (2000a), Dynamique individuelle et reproduction familiale au sein d'une société rurale. Le Pays de Herve dans la seconde moitié du XIX siècle, Liège (Thèse non publiée).

Neven, M. (2000b), "Mortality Differentials and the Peculiarities of Mortality in an Urban-Industrial Population: a Case Study of Tilleur, Belgium", Continuity and change, 15 (2), 297-329.

O'NeILl, B. (1987), Social Inequality in a Portuguese Hamlet. Land, Late Marriage, and Bastardy 1870-1978, Cambridge, New York, New Rochelle, Melbourne, Sydney.

Oris, M., OCHIAI, E. (2000, à paraître), "Family Crisis in the Context of Different Family Systems: Frameworks and Evidence on "When dad died", in When dad died. Individuals and families coping with family stress, R. Derosas, M. Oris, O. Saïto (eds).

OrTMAYR, N. (1995), "Late Marriage: Causes and Consequences of the Austrian Alpine Marriage Pattern", 49-63, in The European Peasant Family and Society. Historical Studies, R. Rudolph (ed.), Liverpool, Liverpool University Press.

Perrenoud, A. (1993), "Mobilité et reproduction à l'échelle d'une communauté rurale ", 449-461, Mesurer et comprendre. Mélanges offerts à Jacques Dupaqquier, Paris, PUF.

Perrenoud, A. (1998), "The Coexistence of Generations and the Availability of Kin in a Rural Community at the Beginning of the Nineteenth Century", The History of the Family. An International Quarterly, 3 (1), 1-15.
Pfister, U. (1994), "Volume et prix sur le marché immobilier de trois commune zurichoises au XVIII e siècle ", 71-94, in Les mouvements longs des marchés immobiliers ruraux et urbains en Europe (XVT-XIX' siècles), M. Dorban, P. Servais (eds), Louvain-laNeuve, Academia.

Ramella, F. (1984), Terra e telai. Sistemi di parentela e manifattura nel Biellese dell'Ottocento, Torino, Einaudi.

REHER, D. (1997), Perspectives on the Family in Spain, Past and Present, Oxford, Clarendon Press.

Rosental, P.-A. (1991), " Pratiques successorales et fécondité: l'effet du Code civil de 1804 ", Économie et Prévision, 100101, 231-238.

RugGles, S. (1996), "The Effects of Demographic Change on Multigenerational Family Structure: United States Whites, 1880-1980", 21-40, in Les systèmes démographiques du passé, A. Bideau, A. Perrenoud, K. A Lynch, G. Brunet (éd.), Lyon, Programme Rhône-Alpes de Recherches Sciences Humaines.

Sabean, D. W. (1990), Property, Production, and Family in Neckerhausen, 1700-1870, Cambridge, Cambridge University Press.

SABEAN, D. W. (1998), Kinship in Neckerhausen, 1700-1870, Cambridge, Cambridge University Press.

Salitot, M. (1988), Héritage, parenté et propriété en Franche-Comté du XIIF siècle à nos jours, Paris, A.R.F. Éditions/L'Harmattan.

Segalen, M. (1978), "Cycle de vie familial et transmission du patrimoine en Bretagne: analyse d'un cas ", Ethnologie française, 4, 271-278.

SEGALEN, M. (1985), Quinze générations de Bas-Bretons. Parenté et société dans le Pays Bigouden Sud 1720-1980, Paris, PUF.

SEgalen, M., Ravis-Giordani, G. (dir.) (1994), Les cadets, Paris, CNRS-Éditions.

SIDDLE, D. J. (1986), "Inheritance Strategies and Lineage Development in Peasant Society", Continuity and Change, 3, 333361. 
Sieder, R., Mitterauer, M. (1983), “The Reconstruction of the Family Life Course: Theoretical Problems and Empirical Results", 309-345, in Family Forms in Historic Europe, R. Wall, J. Robin, P. Laslett (eds), Cambridge, Cambridge University Press.

SkInNeR, G.W. (1999), "Family System and Demographic Processes", 53-95, in Antropological Demography. Toward a New Synthesis, D. I Kertzer., T, Fricke (eds), Chicago, University of Chicago Press.

SMITH, R. (ed.) (1984), Land, Kinship and Life-Cycle, Cambridge, Cambridge University Press.

Thorner, D., Kerblay, B., Smith, R.E.F. (1966), Chayanov on the Theory of the Peasant Economy, Homewood, Illinois.

TODD, E. (1990), L'invention de l'Europe, Paris, Éditions du Seuil.
TODD, E. (1999), La diversité du monde. Famille et modernité, Paris, Éditions du Seuil.

Viazzo, P. P. (1989), Upland Communities: Environment, Population and Social Structure in the Alps since the Sixteenth Century, Cambridge, Cambridge University Press.

WALl, R., LASLETT, P. (eds) (1972), Household and Family in Past Time, Cambridge, Cambridge University Press.

Wegge, S. (1999), “To Part or not to Part: Emigration and Inheritance in Nineteenth-Century Hesse-Cassel", Explorations in Economic History, 36, 30-55.

YVER, J. (1966), Essai de géographie coutumière; égalité entre héritiers et exclusion des enfants dotés, Paris, Sirey.

\section{RÉSUME}

Les tendances les plus récentes de la recherche historique relatives à la reproduction familiale mettent en évidence l'élaboration progressive d'un dialogue interdisciplinaire qui croise les temps des individus, des familles et des communautés. Au cours des dernières années, la démographie historique d'une part, l'histoire de la famille et l'ethnologie d'autre part, ont tissé des liens de plus en plus étroits. La première a concentré son regard sur l'influence des processus démographiques sur les formes familiales, les deuxièmes se sont efforcées d'illustrer l'impact des systèmes familiaux sur les dynamiques démographiques. Les résultats ont amplement illustré la variété des solutions mises en ouvre par les familles pour assurer leur reproduction. Loin de constituer une limite aux possibilités d'interprétation des systèmes familiaux, cette variété représente, au contraire, une véritable richesse, d'autant que l'apport de méthodes nouvelles, parmi lesquelles l'Event History Analysis devrait permettre de mieux évaluer l'impact du court terme, de l'accidentel, sur les stratégies de long terme à l'échelle familiale, c'est-à-dire celles de la transmission entre générations.

\section{SUMMARY}

The most recent trends of historical research relating to family reproduction sheds light on the progressive elaboration of an interdisciplinary dialogue which mixes individuals', families' and communities time. During the last few years, historical demography on the one hand, family history and ethnology on the other hand, have built still narrower links. The first one concentrated itself on the influence of demographic processes on family forms; the second ones tried to illustrate the impact of family system on demographic dynamics. The results largely illustrated the variety of solutions put into effect by families to ensure their reproduction. Far from being a limit for the interpretation possibilities of family systems, this variety represents on the contrary a real wealth and this even more since new methods, among which event history analysis, should make it possible to estimate the impact of short run on strategies on the long run at the family level, i.e. those of transmission between generations. 